\title{
Study on the On/Off Ratio of the Cylindrical Surrounding Gate CNT Transistor Using Nonequilibrium Green's Function Approach
}

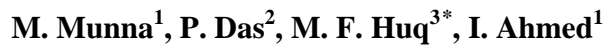 \\ ${ }^{1}$ Department of Electrical and Electronic Engineering, University of Dhaka, Dhaka-1000, \\ Bangladesh \\ ${ }^{2}$ Dept. of Electrical Engineering, University of California, Riverside, California, USA \\ ${ }^{3}$ Department of Nuclear Engineering, University of Dhaka, Dhaka-1000, Bangladesh
}

Received 1 June 2014, accepted in final revised form 01 April 2015

\begin{abstract}
The effects of the nanotube diameter, channel length, gate dielectric constant and gate dielectric thickness on the on-off current ratio performance of cylindrical surrounding gate carbon nanotube transistors are studied using a $\pi$-orbital tight binding simulation model. The focus is both on Schottky barrier and the doped source-drain contact devices. The on current significantly improves with high- $\kappa$ gate dielectric, whereas off current decreases. The device on-off current ratio improves, from $6.33 \times 10^{5}$ to $1.5 \times 10^{6}$ for doped contact and from $0.61 \times 10^{4}$ to $1.22 \times 10^{4}$ for SB device with thinner gate oxide. Minimum leakage current increases with larger diameter tube but on-current has no significant improvement. $\mathrm{I}-\mathrm{V}$ characteristics are independent of channel length when it is larger than $15 \mathrm{~nm}$. Significant increase in off-current occurs due to scaling the channel length down to $10 \mathrm{~nm}$ but on-off ratio still exceeds $10^{3}$. In all cases, on-off ratio is higher and the effect of scaling is more prominent for doped contact devices than SB contact devices.
\end{abstract}

Keywords: Carbon nanotube; Transistor; NEGF; On-off Ratio.

(c) 2015 JSR Publications. ISSN: 2070-0237 (Print); 2070-0245 (Online). All rights reserved. doi: http://dx.doi.org/10.3329/jsr.v7i1-2.19031

J. Sci. Res. 7 (1-2), 11-21 (2015)

\section{Introduction}

A higher integration density has come out as a result of aggressive scaling of CMOS devices over different technology generations. However, scaling of the devices beyond 10 $\mathrm{nm}$ is affected by "short channel effects" such as exponential increase in leakage current and large parameter variations [1]. CMOS will reach its performance limits by 2020, as predicted by the International Technology Roadmap for Semiconductors (ITRS) [2]. Therefore, search for alternatives to MOSFETs in sub-10 nm regime have started through

* Corresponding author: fazlul.huq@du.ac.bd 
research. Single electron transistors [3], silicon nanowire transistors [4, 5], and carbon nanotube field-effect transistors (CNTFETs) [6] have been proposed as candidates for future electron devices. There has been remarkable progress in performance and physical understanding of CNTFET [7] since their first demonstration in 1998 [8]. For low-bias conditions at low temperatures ballistic transport in CNTs has been demonstrated experimentally [9]. High-performance CNT transistors operating close to the ballistic limit has also been reported [10, 11]. Exceptional device characteristics can be expected as the experimentally obtained carrier mobilities are in the order of $10^{4} \mathrm{~cm}^{2} /$ V.s [12]. Significant progress has been made in fabrication process of CNT such as separating the semiconducting and metallic tubes [13] and controllable deposition techniques [14] and CNT thin film deposition [15]. Integration of high- $\kappa$ gate dielectric insulator $[16,17]$ and use of excellent source and drain metal contacts [10] has been demonstrated with improvement in performance. Transistors with exceeding performance to the state of the art, Si MOSFET are achieved. The transistor delivers a current of $\sim 20 \mu \mathrm{Aat}\left|V_{G}-V_{T}\right| \sim$ $1 \mathrm{~V}$ and has a near-ballistic source-drain conductance of $\sim 0.5 \times 4 \mathrm{e}^{2} / \mathrm{h}$.

Two structures have been recommended in literature for CNTFETs. In one structure CNT channel is directly connected with two metal electrodes at both ends. Schottky barriers are formed at the metal electrode junctions. This structure is known as SBCNTFET. This device is operated by modulation of the tunneling barrier at the source contact. The other structure also uses a single-wall CNT as CNT channel. But in this structure a portion of the channel adjacent to the metal electrodes are heavily doped. This device is known as MOSFER-like CNTFET and the transport mechanism is based on modulation of the barrier height of the conduction band between source and channel regions. Conventional CNTFET with lower leakage current along with high on state current at ballistic regime compared to Schottky barrier CNTFETs (SB-CNTFETs) has been reported recently [6].

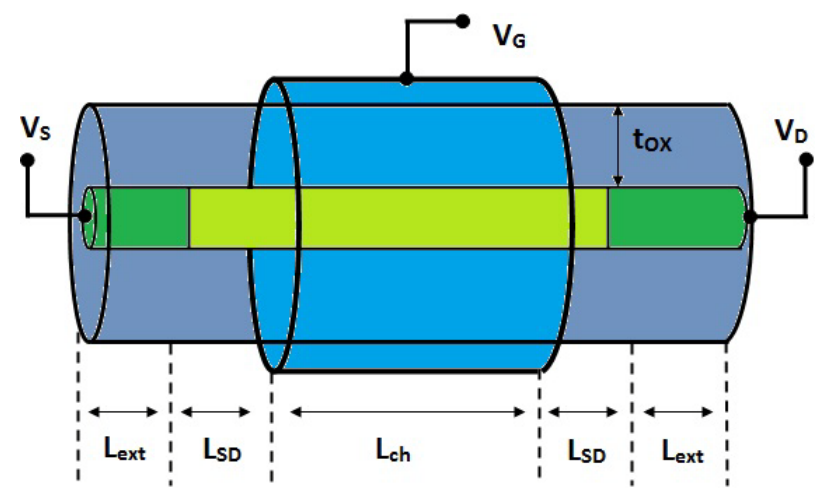

Fig.1. The structure of the device with surrounding gate.

On/off ratio is an important performance parameter of FET. To minimize static power dissipation a low off-current is required. On the other hand, high on-current reduces the gate switching delay. Therefore a high on/off current ratio improves device performance. 
The objective of this research work is to investigate the effect of gate dielectric constant $\kappa$, dielectric thickness $t_{O X}$, diameter $d$, channel length $L_{c h}$ on the on/off ratio of cylindrical gate CNTFET using two simulation tools named CNTFETLab and Cylindrical CNTMOSFET simulator $[18,19]$. Here, we focus on both doped contact device and Schottky barrier contact.

\section{Experimental}

\subsection{CNTFET device structure}

The geometry of the device has source and drain regions $\left(L_{S D}\right)$ and a cylindrical wraparound metallic gate electrode over the intrinsic channel region $\left(L_{c h}\right)$ as shown in Fig. 1. The gate oxide with thickness $t_{O X}$ covers the full length of the tube. $L_{e x t}$ are artificial heavily doped extension regions. These extension regions do not influence the transport in the working part of the transistor but are useful for better numerical convergence purposes. The transistor channel is a $(13,0)$ intrinsic CNT, which results in a band gap of $\sim 0.82 \mathrm{eV}$ and a diameter of $\sim 1.01 \mathrm{~nm}$. The nanotube length is $\sim 20 \mathrm{~nm}$, consisting of $\sim$ $3.9 \times 10^{3}$ carbon atoms. Other device parameters used for the simulation are as follows: source/drain length, $\mathrm{LSD}=30 \mathrm{~nm}$, gate oxide thickness $t_{O X}=2 \mathrm{~nm}\left(\mathrm{HfO}_{2}\right.$ with $\left.\mathrm{k}=16\right)$, the source/drain doping NSD $=1.5 / \mathrm{nm}$. These are the nominal parameters as suggested by Lundstrom et al. [20], used throughout our simulation unless specified otherwise. A selfconsistent Poisson-NEGF simulation in the mode space using the recursive algorithm for computing the Green's function is performed as illustrated in next section.

\subsection{Simulation approach}

Simulation is done by numerically solving the Schrödinger and Poisson equations selfconsistently utilizing nonequilibrium Greens function formalism (NEGF) [21]. $k$-Space approach is used instead of real space to reduce the computational cost [20]. The device Hamiltonian used in this work is based on the atomistic nearest neighbor pz-orbital tightbinding approximation. NEGF modeling of CNTFETs considering ballistic transport is described elsewhere [20, 22].

The retarded Greens function for the device in matrix form is given by,

$G(E)=\left[\left(E+i \eta^{+}\right) I-H_{p z}-\Sigma(E)\right]^{-1}$

Where, $\eta^{+}$is an infinitesimal positive value, and I is the identity matrix. The selfenergy contains contributions from all mechanisms of relaxations, which are the source and drain electrodes, and from scattering,

$\Sigma(E)=\Sigma S(E)+\Sigma D(E)+\Sigma_{\text {scat }}(E)$ 
The description of the Hamiltonian matrix $H_{p z}$ is given in [22, 20]. Under ballistic condition the current from drain to source is given by,

$I=\frac{4 E}{\mathrm{~A}} \int_{-\mathrm{s}=\frac{\mathrm{d}}{2 \pi}}^{+\mathrm{d}} T(E)\left[f\left(E-E_{S}^{B}\right)-f\left(E-E_{D}^{F}\right)\right.$

With the transmission coefficient $T(E)$ given by,

$T(E)=\operatorname{Trace}[\mathrm{TS}(\mathrm{E}) \mathrm{G}(\mathrm{E}) \Gamma \mathrm{D}(\mathrm{E}) \mathrm{G}+(\mathrm{E})]$

Equation (4) is the famous Landauer equation that is widely used in mesoscopic transport [20]. The electrostatic potential and the charge distribution are coupled through the Poissons equation as well, leading to the Poisson NEGF self-consistency requirement. The 2-D Poisson equation for the cylindrical transistor geometry in Fig. 1 is,

$\Delta^{2} U(r, z)=-\frac{\rho\left(r_{z} z\right)}{\varepsilon}$

Here, $\rho(\mathrm{r}, \mathrm{z})$ is the net charge density distribution which includes dopant density as well.

\section{Results and Discussions}

It has been shown that CNTFETs operates essentially ballistic up to several hundred nanometers in length [18]. Since the channel length for CNTFETs of interest is about 10 $\mathrm{nm}$ [19], ballistic transport is assumed through the nanotube. We also restrict our attention to steady-state current-voltage characteristics, which are relevant to high-speed operation of digital circuits [20]. For our investigation we selected zigzag CNT as it is semiconducting.

\subsection{Effect of gate insulator dielectric constant}

First we explored the performance analysis by the use of a high- $\kappa$ coaxial gate insulator. Figs. 2(a) and 2(b) represents the $I_{D}$ vs. $V_{G S}$ characteristics for both doped contact and SB contact respectively at $V_{D}=0.5 \mathrm{~V}$ for four gate insulators, (1) $\kappa=3.9$, (2) $\kappa=8$, (3) $\kappa=14$ (3) $\kappa=25$. Using high- $\kappa$ gate insulator instead of the $\mathrm{SiO}_{2}$ gate insulator improves oncurrent of both Schottky-barrier and doped contact CNT-FET, whereas off current decreases for both cases. But the effect is more prominent in doped contact device than SB contact device. Also the current-voltage characteristics cross for doped CNTFET are at a gate bias of around $0.2 \mathrm{~V}$ for all gate dielectrics. This is because at that bias $\approx E g / 2$ ) the channel potential is approximately in flat band condition with the source fermi level [23]. The scenario can be explained through Figs. 3(a) and 3(b). At the on-state, the charge in the nanotube channel can produce a considerable self-consistent potential which raises the conduction band in the CNT body and increases barrier for electron at source 
end. When a high- $\kappa$ gate insulator is used, the electric field produced by the charge on the tube is effectively screened by the high- $\kappa$ gate insulator, and the self-consistent potential is smaller. Compared to $\mathrm{SiO}_{2}$ gate insulator the barrier at the source contact is lower and the conduction band in the CNT body is more effectively lifted down by the gate voltage by applying $\mathrm{HfO}_{2}$ gate insulator as shown in Fig. 3(a). The barrier at the source contact is lower and the conduction band in the CNT body is more effectively lifted down by the gate voltage by applying $\mathrm{HfO}_{2}$ gate insulator. Hence it is evident that, High- $\kappa$ gate insulators afford high capacitance without relying on ultra-small film thickness, thus allowing for efficient charge injection into transistor and meanwhile reducing directtunneling leakage currents.

The reason for such behavior in case of Schottky barrier is as follows. Fig. 3(b), which represents the conduction band profiles, for three insulators at the on-state, sheds light on these results. Due to thin gate oxide the Schottky barriers are thin. So the charge density inside the tube is high. When the gate dielectric constant is low, this charge produces a considerable self-consistent potential. As a result the conduction band floats up, which makes the Schottky barrier thicker and the conduction band in the interior of the channel higher. As the tunneling current exponentially depends on the Schottky barrier thickness, the on-current of transistors with low gate dielectric constants is much smaller. Converse is true for high gate dielectric constant oxide materials.

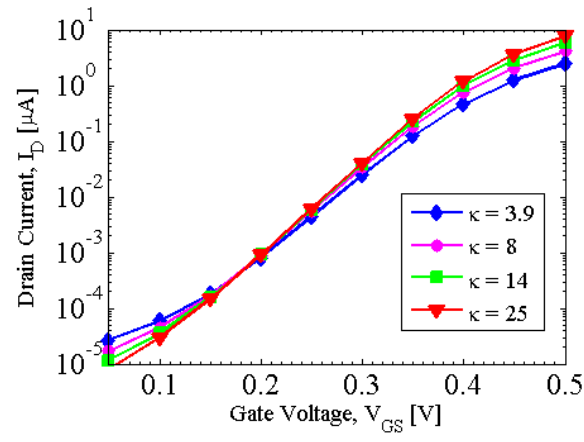

(a) Doped contact

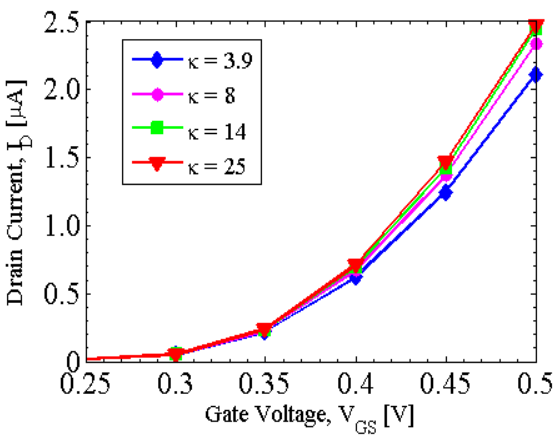

(b) SB contact

Fig. 2. The variations of drain current against gate voltage for different values of gate dielectric constant.

\subsection{Effect of oxide thickness}

The on/off current ratio for different oxide thickness is shown in Figs. 4(a) and 4(b) for both doped contact and SB contact devices respectively. The on/off current ratio improves with thinner oxide. The on/off current ratio improves from $6.33 \times 10^{5}$ to $1.5 \times 10^{6}$ for the doped contact devices and from $6.1 \times 10^{3}$ to $1.22 \times 10^{4}$ for SB contact devices when the gate oxide thickness changes from 4.5 to $2 \mathrm{~nm}$. The doped contact devices have higher 
on/off current ratio and the $t_{O X}$ effects on on/off current ratio is relatively higher in doped contact devices. Shaahin et al. also reported on/off ratio for doped contact device with chirality $n=13$ is in the order of $10^{6}$ [24]. For thinner oxide thickness total capacitance will increase. So for same gate bias, with thinner oxide the charge in the channel will increase. Consequently conduction band in the channel will be lowered, therefore source to channel barrier will decrease. This is the reason on-current increases with thinner oxide, eventually on/off ratio increases.

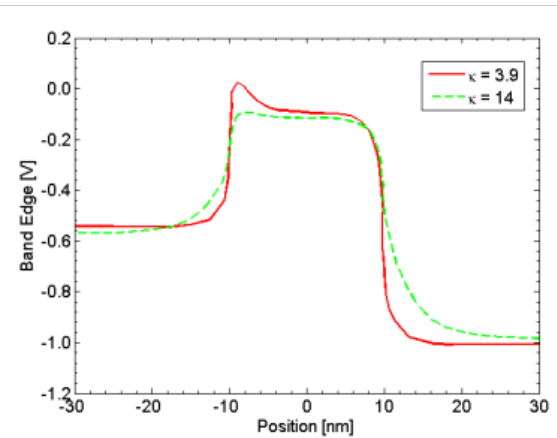

(a) Doped contact

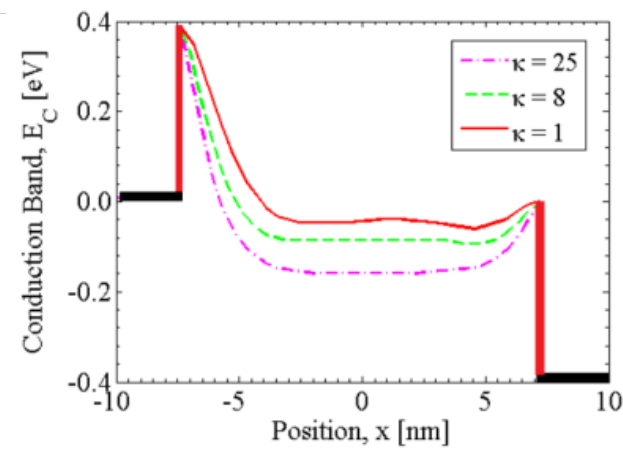

(b) SB contact

Fig. 3. Conduction band profile at different dielectric constants.

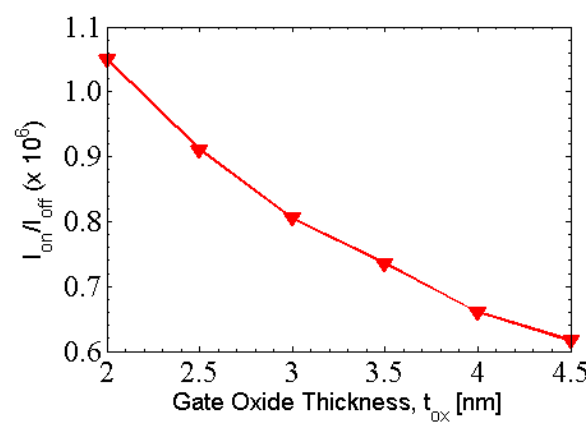

(a) Doped contact

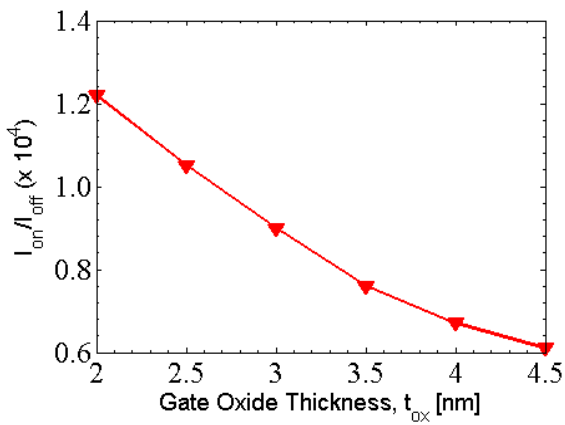

(b) SB contact

Fig. 4. Variations of on/off ratio against gate oxide thickness, $\mathrm{t}_{\mathrm{Ox}}$.

\subsection{Effect of diameter}

The impact of diameter on the I-V characteristics of doped CNTFET is investigated in detail. We compared it with the I-V characteristics for SB CNTFET reported by Guo et al. [25]. From Figs. 5(a) and 5(b) it is evident that diameter has little effect on the on-current of the device, but with decreasing diameter off-current drastically reduces. Consequently 
on/off current ratio increases with reduction of diameter which is shown in Figs. 6(a) and 6(b) for doped and SB contact respectively.

There is an inverse relation between channel diameter, i.e., " $n$ " value of the channel and its energy bandgap. For higher " $n$ " values the valance band edge of channel is closer to the conduction band edge of the source at the low gate bias than lower " $n$ " values for doped contact devices [26]. Consequently band to band tunneling leakage current increases in off state. On the other hand in on-state separation between source conduction band and channel valance band is high. As a result there is almost no tunneling in this case and dominant mechanism for electron transport is thermionic emission [26]. For Schottkybarrier contact using a large diameter tube reduces the band gap and significantly increases the minimum leakage current at the ambipolar bias point. At the same time, the on-current is also improved, but the on/off current ratio decreases significantly as the nanotube diameter increases.

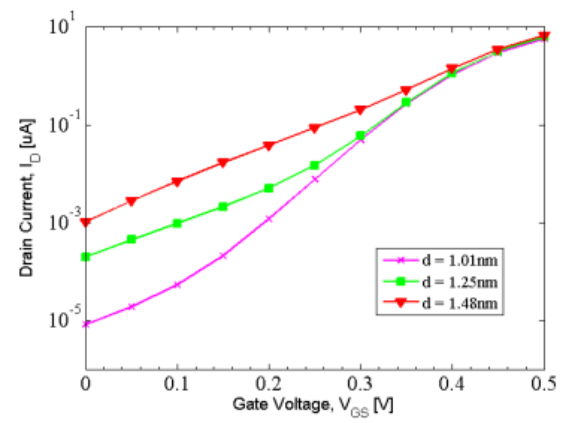

(a) Doped contact

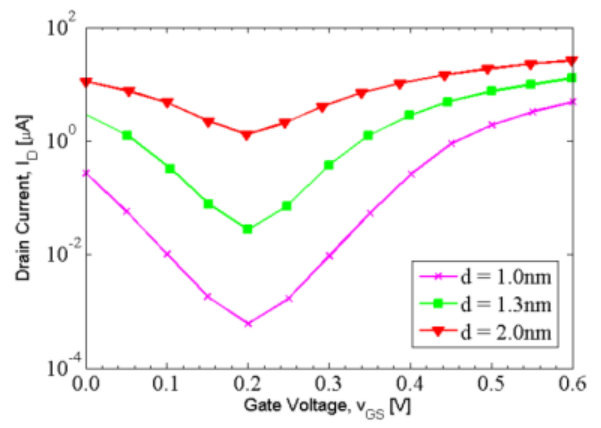

(b) SB contact [24]

Fig. 5. Drain current dependence on gate voltage for different diameters.

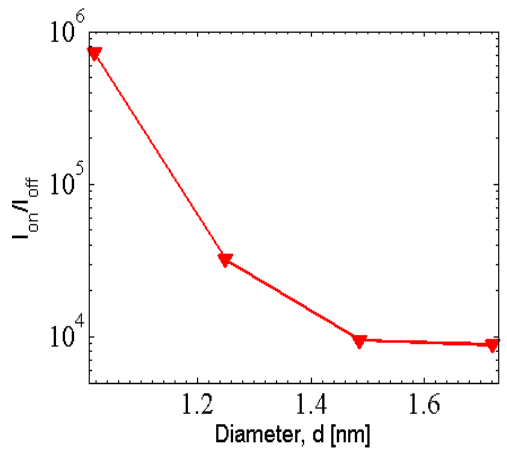

(a) Doped contact

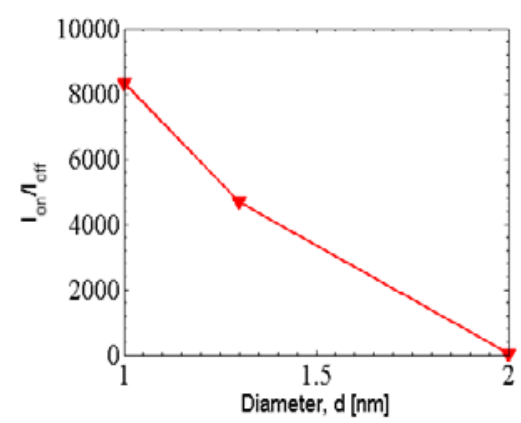

(b) SB contact

Fig. 6. The dependence of on/off ratio on channel diameter. 


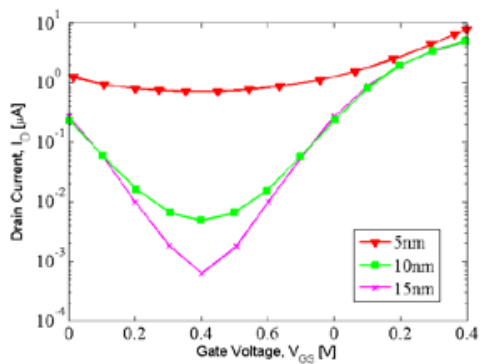

(a) Doped contact

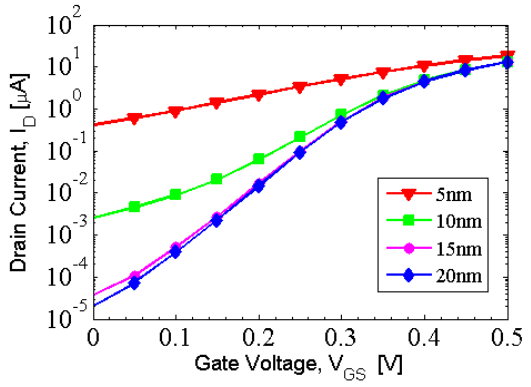

(b) SB contact [25]

Fig. 7. Variations of drain current against gate voltage for different channel lengths.

From Fig. 6 it is evident that the on/off current ratio decreases more rapidly for doped contact device than SB device, with diameter. This is due to the fact that while the on current remains almost same but off current increases more significantly with diameter as depicted.

\subsection{Effect of Channel Length}

Fig. 7 explores the issue of channel length scaling for doped contact and Schottky barrier CNTFET. In order to establish the ultimate scaling limit imposed by source-drain tunneling for CNTFET, very thin gate oxide $\left(t_{O X}=2 \mathrm{~nm}\right)$ is used to ensure excellent gate control electrostatics. We have found that for doped contact device when the channel length is longer than $15 \mathrm{~nm}$, the I-V characteristics are independent of the channel length (as shown in Fig. 7(a)) because the channel is ballistic and effective source to drain barrier is thick and there is no significant tunneling. Scaling the channel length down to10 nm significantly increases the off-current, but the on/off current ratio still exceeds $10^{4}$, which is excellent for digital logic. If the channel length is aggressively scaled down to $5 \mathrm{~nm}$, the on-off current ratio decreases to less than100 due to significant source-drain quantum tunneling. Fig. 8 explains the reason in case of doped contact CNTFETs. It shows conduction band profile for three different channel lengths. We see that when $L_{c h}=30 \mathrm{~nm}$, the effective source to channel barrier is larger. When channel length decreases to $L_{c h}=15$ $\mathrm{nm}$, the barrier decreases only slightly. But when $L_{c h}=5 \mathrm{~nm}$ barrier decreases significantly, so there is a large amount of tunneling current and off current increases significantly. From Fig. 7(b) it is evident that Schottky barrier CNTFET shows similar I-V characteristics as doped contact but the change in on/off current ratio is less. Figs. 9(a) and 9(b) shows the on/off current ratio for doped and Schottky barrier CNTFET respectively. It is observed that channel length has more significant effect on on/off ratio for doped contact than Schottky barrier contact devices.

In all cases on-off ratio is higher and the effect of scaling is more prominent for doped contact devices than SB contact devices. On-off ratio could be tuned upto $10^{6}$ and $10^{4}$ order of magnitude for doped contact and Schottky barrier contact devices respectively. 
Lin et. al. demonstrated multiple tube CNTFET with on/off ratios of $5 \times 10^{3}$ up to $5 \times$ $10^{5}$ and threshold voltage up to approximately 2V [27]. Sangwan et al. demonstrated percolating networks of metallic and semiconducting carbon nanotubes FET with mobility up to $50 \mathrm{~cm}^{2} / \mathrm{Vs}$ at on/off ratio $>10^{3}$ obtained at channel width $\mathrm{W}=50 \mu \mathrm{m}$ and $L>70$ $\mu \mathrm{m}$ for $p=0.54-0.81 \mathrm{CNT} \mathrm{s} / \mu \mathrm{m}^{2}$ [28]. Sun et al. demonstrated carbon nanotube thin film transistors with mobility of $35 \mathrm{~cm}^{2} \mathrm{~V}^{-1} \mathrm{~s}^{-1}$ and an on/off ratio of $6 \times 10^{6}$ [29]. Khondaker et al. demonstrated semiconducting enriched carbon nanotube align arrays FET with on/off ratio as high as approximately $\approx 10^{4}$ at semiconducting enriched (99\%) nanotube density approximately $\approx 1$-SWNTs/ $\mu$ m [30]. These experimental evidences closely support our analysis.

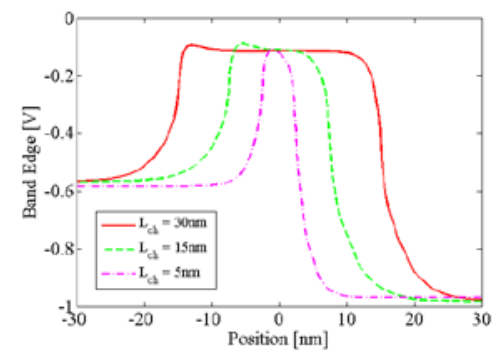

Fig. 8. Conduction band profile for nominal CNTFET at different channel lengths.

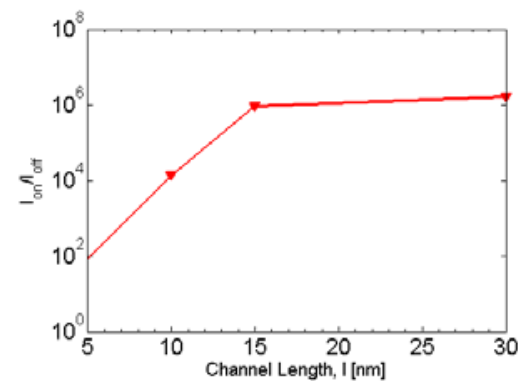

(a) Doped contact

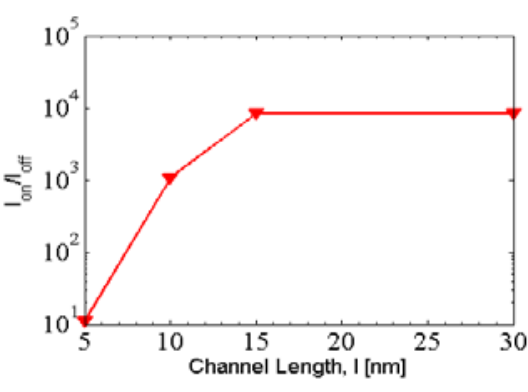

(b) SB contact

Fig. 9. Variations of on/off ratio against different channel lengths.

\section{Conclusion}

A study of the effect of different scaling of cylindrical surrounding gate CNT transistors is performed using a $\pi$ - orbital tight binding model. The impacts of gate dielectric constant, thickness, annotate diameter and channel length are investigated through simulating the behavior of the device depending on these parameters. The result of the simulation study clearly indicates that for optimum configuration of device parameters, the on/off ratio of doped contact device and Scotty barrier device has the potentials to be tuned up to $10^{6}$ and $10^{4}$ order of magnitude respectively. The on-off ratio behavior of doped contact and Schottky barrier CNTFETs for varying device parameters has been summarized in Tables 
1 and 2 respectively. The behavioral trends of doped contact and Schottky barrier devices from the tables can be used as a reference to choose optimum configuration of device parameters which being the most important issue to fabricate a high performance CNTFET with good scaling potential.

Table1. Parameter dependent behavior of doped contact CNTFET

\begin{tabular}{|c|c|c|c|c|c|}
\hline Prop & Change & $\mathrm{On} \mathrm{Cu}$ & Off C & tratio & $\begin{array}{c}\text { Remarks for high } \\
\text { performance }\end{array}$ \\
\hline $\begin{array}{c}\text { Dielectric } \\
\text { Constant, } \kappa\end{array}$ & $\mathrm{e}$ & $\begin{array}{l}\text { Increases more } \\
\text { rapidly }\end{array}$ & Dec & rapidly & High $\kappa$ dielectric \\
\hline $\begin{array}{c}\text { Oxide } \\
\text { Thickness, }\end{array}$ & Decrease & Increases & $\begin{array}{r}\text { Remair } \\
\text { sa }\end{array}$ & $\begin{array}{r}\text { Increa } \\
\text { ra }\end{array}$ & Thi \\
\hline Dian & $\mathrm{D}$ & $\begin{array}{l}\text { Rema } \\
\text { almost }\end{array}$ & Decreases & $\begin{array}{l}\text { Increases more } \\
\text { rapidly }\end{array}$ & $\begin{array}{l}\text { Lower diameter } \\
\text { Tube }\end{array}$ \\
\hline $\begin{array}{l}\text { Channe } \\
\text { length, L }\end{array}$ & Decrease & $\begin{array}{l}\text { Remains } \\
\text { almost same }\end{array}$ & hcreases & Decreases & $\begin{array}{c}\text { Could be less than } \\
10 \mathrm{~nm}\end{array}$ \\
\hline
\end{tabular}

Table 2. Parameter dependent behavior of Schottky barrier CNTFET

\begin{tabular}{|c|c|c|c|c|c|}
\hline Properties & Change & On Current, $\mathrm{I}_{\mathrm{on}}$ & Off Current, $\mathrm{I}_{\text {off }}$ & On/off current ratio & $\begin{array}{c}\text { Remarks for high } \\
\text { performance }\end{array}$ \\
\hline $\begin{array}{c}\text { Dielectric } \\
\text { Constant, } \kappa\end{array}$ & ncrease & Increases & $\begin{array}{l}\text { No significant } \\
\text { decrease }\end{array}$ & Increases & High $\kappa$ dielectric \\
\hline $\begin{array}{c}\text { Oxide } \\
\text { Thickness, } \mathrm{t}_{\mathrm{ox}}\end{array}$ & Decrease & Increase & $\begin{array}{c}\text { Remains almost } \\
\text { same }\end{array}$ & Incre & Thinner oxide \\
\hline Diameter, d & Decrease & Little increase & Dec & Incr & $\begin{array}{l}\text { Lower diameter } \\
\text { Tube }\end{array}$ \\
\hline $\begin{array}{l}\text { Channel } \\
\text { length, } \mathrm{L}_{\mathrm{ch}}\end{array}$ & Decrease & $\begin{array}{l}\text { Remains } \\
\text { almost same }\end{array}$ & Increases & Decreases & $\begin{array}{c}\text { Should be larger } \\
\text { than 10nm }\end{array}$ \\
\hline
\end{tabular}

On-off ratio increases for all the parameter scaling except channel length and the effect of scaling is relatively higher for doped contact devices. However, for a complete evaluation of the performance, effect on transconductance, unity current gain frequency, static and dynamic power dissipation should be studied.

\section{Acknowledgment}

The authors would like to thank Jing Guo \& Neophytos Neophytou for the simulator softwares. They would also like to thank Network for Computational Nanotechnology (NCN) for the java engine used to run the simulator.

\section{References}

1. K. Roy, S. Mukhopadhyay, and H. Mehmoodi-Meimand, IEEE Proc. 91, 305327 (2003).

2. C.Macilwain, Nature, 436, 22 (2005). http://dx.doi.org/10.1038/436022a

3. D.L. Klein, R. Roth, A.K.L. Lim, A.P. Alivisatos, and P.L.McEuen, Nature 389, 699701 (1997). 
4. G. Zheng, W. Lu, S. Jin, and C.M. Lieber, Adv. Mater. 16, 18901893 (2004).

5. J. Goldberger, A.I. Hochbaum, R. Fan, and P. Yang, Nano Lett. 6, 973 (2006). http://dx.doi.org/10.1021/nl060166j

6. J. Guo and M. S. Lundstrom, 'Carbon Nanotube Electronics', edited by A. Javey and J. Kong, (Springer, New York, 2009).

7. M.P. Anantramand and F. Leonard, Rep. Prog. Phys. 69, 507561 (2006).

8. R. Martel, T. Schmidt, H. R. Shea, T. Hertel, and P. Avouris, Appl. Phys. Lett. 73, 2447 (1998). http://dx.doi.org/10.1063/1.122477

9. J. Kong, E. Yenilmez, T.W. Tombler, W. Kim, and H. Dai, Phys. Rev. Lett. 87, 106801 (2001). http://dx.doi.org/10.1103/PhysRevLett.87.106801

10. A. Javey, J. Guo, Q. Wang, M. Lundstrom, and H. Dai, Nature 424, 654657 (2003). http://dx.doi.org/10.1038/nature01797

11. A. Javeyetal, Nano Lett. 4, 13191322 (2004).

12. X. Zhou, J.Y. Park, S. Huang, J. Liu, and P. L. McEuen, Phys. Rev. Lett. 95, 146805 (2005). http://dx.doi.org/10.1103/PhysRevLett.95.146805

13. S. Arnold, A.A. Green, J.F. Hulvat, S.I. Stupp, and M.C. Hersam, Nature Nanotechnology $\mathbf{1}$, 6065 (2006). http://dx.doi.org/10.1038/nnano.2006.52

14. R. Krupke, F. Hennrich, H. vonLohneysen, and M.M. Kappes, Science 301, 344 (2003). http://dx.doi.org/10.1126/science.1086534

15. B. Ahmmad, Y. Kusumoto, M. Abdulla-Al-Mamun, A. Mihata, and H. Yang, J. Sci. Res. 1, 430 (2009).

16. A. Javey, H. Kim, M. Brink, Q. Wang, A. Ural, J. Guo, P. McIntyre, P. McEuen, M. Lundstrom, and H.J. Dai, Nature Materials 1, 241 (2002). http://dx.doi.org/10.1038/nmat769

17. S.J. Wind, J. Appenzeller, R. Martel, V. Derycke, and P. Avouris, J. Vac. Sci. Technol. B, 20, 2798 (2002).

18. N. Neophytou, S.S. Ahmed, E. Polizzi, G. Klimeck, M. Lundstrom, 'CNTFET Lab', http://nanohub.org/resources/cntfet, DOI:10.4231/D32B8VB3H (2012).

19. G.W. Budiman, Y. Gao, X. Wang, S. Koswatta, M. Lundstrom, 'Cylindrical CNTMOSFET Simulator', http://nanohub.org/resources/moscntr, (2010).

20. S.O. Koswatta, S. Hasan, M.S. Lundstrom, Electron Devices, IEEE Transactions on 54, 2339, (2007).

21. S. Datta, Electronic Transport in Mesoscopic Systems (Cambridge University Press, NewYork, 1995).

22. J. Guo, S. Datta, M. Lundstrom, and M.P. Anantam, Int. J. Multiscale Comput. Eng. 2, 257, (2004). http://dx.doi.org/10.1615/IntJMultCompEng.v2.i2.60

23. K. Alam and R.K. Lake, J. Appl. Phys. 100, 024317 (2006). http://dx.doi.org/10.1063/1.2218764

24. S.G. Shirazi and S. Mirzakuchaki, Nanoelectronics Conference (INEC), $3^{\text {rd }}$ International IEEE, (2010).

25. J. Guo, S. Datta, and M. Lundstrom, IEEE Transactions on Electron Devices 51, 172 (2004). http://dx.doi.org/10.1109/TED.2003.821883

26. S.G. Shirazi and S. Mirzakuchki, Appl. Phys. Lett. 99, 263104 (2011). http://dx.doi.org/10.1063/1.3672220

27. A. Lin, N. Patil, K. Ryu, A. Badmaev, L. G. De Arco, C. Zhou, S. Mitra, and H. -S. P. Wong, IEEE transactions on nanotechnology 8, 4 (2009). http://dx.doi.org/10.1109/TNANO.2008.2004706

28. V.K. Sangwan, A. Behnam, V.W. Bellarotto, M.S. Fuhrer, A. Ural, and E.D. Williams, Appl. Phys. Lett. 97, 043111 (2010). http://dx.doi.org/10.1063/1.3469930

29. D. Sun, M. Y. Timmermans, Y. Tian, A. G. Nasibulin, E. I. Kauppinen, S. Kishimoto, T. Mizutani, and Y. Ohno, Nature Nanotechnol. 6, 156, (2011). http://www.nature.com/doifinder/10.1038/nnano.2011.1

30. K. Biddut, S. Sarker, S. Shekhar, and S. I. Khondaker., ACS Nano 5, 6297 (2011). http://dx.doi.org/10.1021/nn201314t 